\title{
The mechanism of texture formation during film growth: The roles of preferential sputtering and shadowing
}

\author{
Feng Ying, Richard W. Smith, and David J. Srolovitz \\ Department of Materials Science and Engineering, The University of Michigan, Ann Arbor, \\ Michigan 48109-2136
}

(Received 9 February 1996; accepted for publication 4 September 1996)

\begin{abstract}
Nonequilibrium molecular dynamics simulations have been employed to develop a mechanistic model for the development of an out-of-plane (fiber) texture in polycrystalline thin films. The depositing atoms preferentially sputter film atoms from grains with high surface energies. As the film grows, an atomic shadowing mechanism leads to the lateral growth of the grains with a height advantage - eventually leading to the occlusion of randomly oriented grains. (c) 1996 American Institute of Physics. [S0003-6951(96)01246-6]
\end{abstract}

Vapor deposited, polycrystalline, metallic thin films are used in a wide variety of applications including interconnects in microelectronic devices, magnetic recording underlayers, and corrosion resistant protective coatings. ${ }^{1}$ Nearly all vapor deposited metallic films exhibit a strong out-of-plane crystallographic texture. The texture often strongly influences film performance. Body centered cubic (bcc) films typically exhibit $\langle 110\rangle$ out-of-plane orientations, while face centered cubic (fcc) films commonly have $\langle 111\rangle$ textures. ${ }^{2,3}$ These fiber (strong out-of-plane/random in-plane) textures have been observed in films deposited in thermal evaporation, sputtering and ion beam assisted deposition environments with the degree of texture depending on both temperature and the energy of the particles striking the film surface. Kawanabe et al. ${ }^{4}$ recently showed that the $\langle 110\rangle$ component of the texture of $\mathrm{Cr}$ films decreases with decreasing deposition kinetic energy during normal incidence sputter deposition. Knorr and $\mathrm{Lu}^{5}$ reported a shift from epitaxial growth to $\langle 111\rangle$ fiber texture with increasing self-ion energy in $\mathrm{Cu}$ on $\mathrm{Si}$ grown by the partially ionized beam technique.

The commonly accepted mechanism for the formation of fiber texture is the evolutionary selection model, put forth in the late 1960's by Van der Drift. ${ }^{6}$ In this model, fiber texture results from the difference in growth rates between different crystal faces of the grains on the surface of the film. Grains oriented with their faster growing directions perpendicular to the surface are preserved while slower growing grains are terminated as they intersect the column walls of taller grains. This growth preference may be related to the thermodynamic preference of deposited atoms to condense on low, rather than high-energy surfaces in order to minimize the surface energy. ${ }^{7}$ This is consistent with the observation that the commonly observed out-of-plane orientations correspond to the lowest-energy surfaces.

An alternative to this classical view, is that preferential growth may be affected by differences in sputtering rates from one crystal face to another (see, e. g., Ref. 8). While clearly important in environments where relatively highenergy species are present, this process may contribute significantly to the observed fiber texture during relatively lowenergy sputter and ion-assisted deposition. Previous work has shown that preferential sputtering can lead to the formation of texture in films bombarded with relatively high- energy ion beams at oblique angles. ${ }^{9}$ (By sputtering we mean the ejection of atoms from the growing film surface and not the ejection of atoms from a target material in a plasma-as in typical sputter deposition systems.) In the present work, we present a detailed mechanistic understanding of how preferential sputtering of atoms off the surface by the atoms which are being deposited can lead to a growth advantage for low sputtering rate grain orientations. We then demonstrate the mechanism by which the height advantage leads to the formation of an out-of-plane (fiber) texture.

In order to investigate the influence of the kinetic energy of the particles on sputtering from film surfaces, computer simulations were performed using a two dimensional, nonequilibrium molecular dynamics technique (see Ref. 10 for details). In short, atom trajectories are determined by integrating Newton's equation of motion forward in time, where the force on any atom is solely a result of interaction with other, nearby atoms. The forces are computed as the negative gradient of the familiar Lennard-Jones, pairwise interatomic potential: $\phi\left(r_{i j}\right)=\epsilon\left[\left(\sigma / r_{i j}\right)^{12}-2\left(\sigma / r_{i j}\right)^{6}\right]$, where $\phi_{i j}$ is the energy associated with the bond between atoms $i$ and $j$ separated by a distance $r_{i j}$ and $\epsilon$ and $\sigma$ are parameters that characterize the potential well depth and atomic spacing, respectively. All atoms are assumed to be of the same species. The computational cell is periodic in the direction normal to the incident atom flux, (i.e., in the $X$ direction). The substrate consists of several layers of the equilibrium triangular lattice crystal structure and depositing atoms impinge on the growing film at normal incidence (i.e., the depositing atoms follow straight paths in the negative $Y$-direction). The sample is maintained at a constant temperature via the thermostating operation described in Ref. 11.

Sputtering simulations were performed on single-crystal samples of roughly 40 lattice constants in width (along $X$ ). Substrates with crystallographic orientations of $0^{\circ}$ and $30^{\circ}$ (i.e., the [11] crystallographic axis parallel or at $30^{\circ}$ to $X$ ) were used. The simulations were performed with a substrate temperature of $0.22 \epsilon / k$ ( $k$ is Boltzmann's constant) and monoenergetic deposition kinetic energies between 60 and $200 \epsilon$ at a deposition rate of 0.08 atoms $/ \tau$, where $\tau$ is the time unit, $\tau=\left(M \sigma^{2} / \epsilon\right)^{1 / 2}$, and $M$ is the atom mass. For reference, the melting point of the triangular lattice at zero pressure is $0.416 \epsilon / k$ (Ref. 12) and for a $\mathrm{Cu}$ atom these kinetic energies 


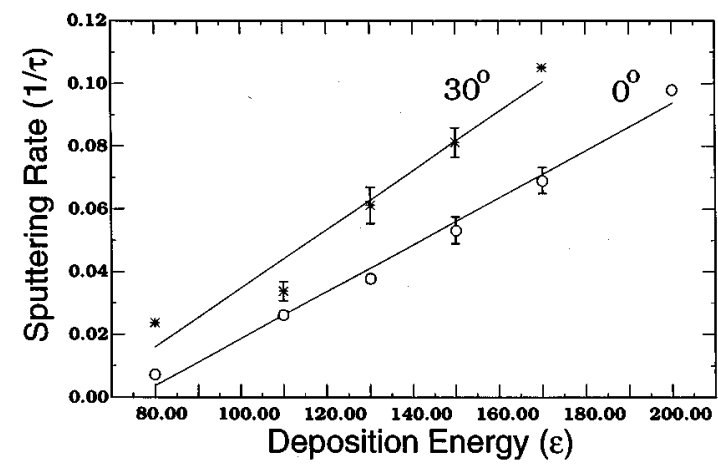

FIG. 1. Sputtering rate (number of atoms ejected or reflected from the surface per unit time) versus kinetic energy of the impinging atoms for crystals with nominal $0^{\circ}$ and $30^{\circ}$ surface orientations.

are in the range of $35-117 \mathrm{eV}$. Throughout each simulation, the number of atoms sputtered or reflected from the surface was measured as a function of time. The slope of the straight line fit through this data yields the sputtering rate for each crystallographic orientation and deposition kinetic energy. Sputtering rate is plotted as a function of deposition kinetic energy for each of the two orientations in Fig. 1. Each data point in Fig. 1, represents an average over five simulations. The sputtering rate increases linearly with increasing deposition kinetic energy for both film orientations. At each energy, the sputtering rate for the $30^{\circ}$ oriented film is larger than for the $0^{\circ}$ oriented film.

The sputtering yield theory put forth by Sigmund, ${ }^{13}$ suggests that the sputtering rate from any given surface is expected to be proportional to the ratio of the kinetic energy of the incident particles over the atomic binding energy at the surface. This is consistent with the results presented in Fig. 1 , where the sputtering rate was found to be proportional to the deposition kinetic energy. Furthermore, the ratio of the sputtering rates from the two different surfaces at fixed deposition kinetic energy is expected to be equal to the inverse of the ratio of the binding energies of the two surfaces. If the binding energies of the $0^{\circ}$ and $30^{\circ}$ surfaces are computed from simple bond counting arguments ( 4 bonds for $0^{\circ}$ and 3 bonds for $30^{\circ}$, assuming perfectly coordinated surface atoms) then a value of $3 / 4=0.75$ is obtained for the ratio of the $30^{\circ}$ and $0^{\circ}$ surface binding energies. This compares favorably with the inverse ratio of sputtering yields of 3.0/3.8 $\approx 0.79$ determined from the slopes of the sputtering curves in Fig. 1.

These results demonstrate that the crystallographic orientation of the film relative to the deposition flux controls the rate at which particles are sputtered from the surface. This suggests that in a polycrystalline film, those grains which are oriented such that their sputtering rate is low will grow faster than the other grains. We expect that this will lead to the development of textures which are dominated by grains of low sputtering rate orientations. The data in Fig. 1 and Sigmund's ${ }^{13}$ sputtering theory suggest that these orientations are those with close-packed surfaces. This is consistent with experimental observations. ${ }^{2,3}$

While the present results suggest that preferential sputtering leads to decreased growth rates, we have not demonstrated that such decreased growth rates lead to the formation

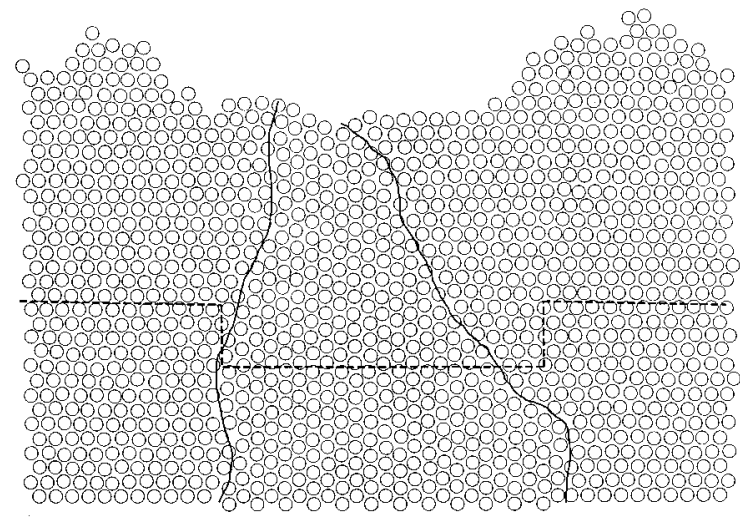

FIG. 2. Typical film grown on a periodic bicrystal substrate in which the grain with a $0^{\circ}$ surface orientation is given an initial height advantage of five lattice constants. The dashed line shows the initial profile of the substrate surface. The solid lines depict the grain boundaries, showing the elimination of the height disadvantaged grain as the film grows in height.

of textured films. The simulations have shown, however, that preferential sputtering will lead to crystallographic orientation dependent height advantage for certain grains. Therefore, we have performed a series of simulations to investigate how a height advantage translates into texture development.

We have performed a series of simulations of film growth with a periodic grain structure consisting of $0^{\circ}$ and $30^{\circ}$ oriented grains (see Fig. 2). Simulations were performed in which either the $0^{\circ}$ or the $30^{\circ}$ oriented grains have height advantages of 2, 5, and 10 atomic layers. Atoms were deposited on these initial structures at a temperature of $0.22 \epsilon / \mathrm{k}$ and a deposition kinetic energy of $0.6 \epsilon$, which is too low to produce an appreciable sputtering rate in the present simulations. As shown in the example in Fig. 2, the film develops in such a fashion that the grains with a height advantage grow laterally (i.e., in the plane of the film) at the expense of the lower grain during the course of film growth. After a finite period of time, the initially lower grains are completely pinched off by the grains that enjoyed the initial height advantage. The results of the entire series of simulations are tabulated in Table $I$ in terms of the ratio of the number of simulations in which the grains with the initial height advantage pinch off the lower grains to the total number of simulations performed. These data show, that the rate at which the grains with the height advantage pinch off the other grains increases with increasing initial height advantage. In none of the simulations performed were the grains with the initial height advantage pinched off by the surrounding grains. The data in this table also demonstrate that the grains

TABLE I. Ratio of the number of independent simulations in which the shorter grain is completely pinched off within 60 lattice constants of the substrate to the total number of simulations performed. $\Delta h$ is the initial height advantage in units of lattice constants. The $0^{\circ}$ and $30^{\circ}$ columns indicate the orientation of the grain which was given the initial height advantage.

\begin{tabular}{rll}
\hline \hline$\Delta h$ & $0^{\circ}$ & $30^{\circ}$ \\
\hline 2 & $6 / 9$ & $3 / 7$ \\
5 & $6 / 6$ & $5 / 6$ \\
10 & $4 / 4$ & $4 / 4$ \\
\hline \hline
\end{tabular}




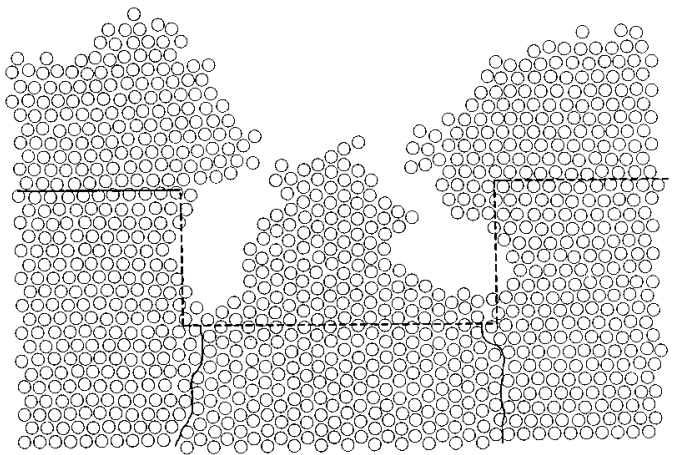

FIG. 3. Typical film grown on a periodic bicrystal substrate with large initial height advantage (ten lattice constants). The self-shadowing mechanism causes voids to nucleate at the bottoms of the steps.

$\left(0^{\circ}\right.$ or $\left.30^{\circ}\right)$ that initially enjoyed the height advantage tend to pinch off those grains without the height advantage regardless of the crystallographic orientation of the grains with the initial height advantage. This result demonstrates that texture development in these films is not a result of surface energy differences.

The mechanism by which grains with a height advantage grow laterally is related to the well-known phenomenon of shadowing, even though deposition is occurring at normal incidence. An atom that is being deposited very close to, but on the low side of a vertical step, is pulled toward the step edge due to the attractive part of the interatomic potential. This gives the depositing atom a component of velocity in the direction toward the riser of the vertical step. Such atoms condense onto the riser, thereby giving it a net growth rate in the outward direction which causes the taller grain to grow at the expense of its neighbor. As this lateral growth continues, the taller grain begins to physically shadow the lower grain. If there is sufficient atomic mobility along the surface, this simply leads to the pinching off of the lower grain. However, if the atomic mobility is low, this can lead to the formation of an overhang, which can both pinch off the lower grain and lead to the incorporation of a void, as shown in Fig. 3.
In conclusion, we have proposed a mechanism in which preferential sputtering of atoms from surfaces of different crystallographic orientation leads to height advantages for grains with low energy surfaces as compared with randomly oriented grains. This height advantage results in the occlusion of the randomly oriented grains by those with low surface energies via a shadowing mechanism. The length of time or, alternatively, the film thickness at which this process leads to a well-formed fiber texture increases with decreasing incident atom kinetic energy (i.e., sputtering rates) and increasing temperature (i.e., diffusional surface smoothing which opposes shadowing). Note that this mechanism will generally lead to the preferential formation of textures corresponding to low surface energies. This occurs simply because the sputtering yield is proportional to the surface energy.

The authors gratefully acknowledge stimulating discussions with Professor S. M. Yalisove and Dr. A. Malhotra. This research was supported by the U.S. Advanced Projects Research Agency and the U. S. Air Force Office of Scientific Research under MURI Grant No. F49620-95-1-0524.

${ }^{1}$ W. D. Nix, Metall. Trans. A 20, 2217 (1989).

${ }^{2}$ L. Tang and G. Thomas, J. Appl. Phys. 74, 5025 (1993).

${ }^{3}$ D. B. Knorr and D. P. Tracy, Appl. Phys. Lett. 59, 3241 (1991).

${ }^{4}$ T. Kawanabe, J. Park, and M. Naoe, Mater. Sci. Eng. A 134, 1305 (1991).

${ }^{5}$ D. B. Knorr and T.-M. Lu, Textures Microstruct. 13, 155 (1991).

${ }^{6}$ A. Van der Drift, Philips Res. Rep. 22, 267 (1967).

${ }^{7}$ M. B. Stearns, C. H. Lee, C.-H. Chang, and A. K. Petford-Long, in $\mathrm{Me}-$ tallic Multilayers and Epitaxy, edited by M. Hong, S. Wolf, and C. C. Gubser (The Metallurgical Society, Warrendale, PA, 1988), p. 55.

${ }^{8}$ E. S. Machlin, Materials Science in Microelectronics (Giro, New York, 1995), p. 83

${ }^{9}$ R. M. Bradley, J. M. E. Harper, and D. A. Smith, J. Appl. Phys. 60, 4160 (1986).

${ }^{10}$ R. W. Smith and D. J. Srolovitz, J. Appl. Phys. 79, 1448 (1996).

${ }^{11}$ M. P. Allen and D. J. Tildesley, Computer Simulation of Liquids (Clarendon, Oxford, 1987).

${ }^{12}$ S. M. Park, S. Kim, I. K. Schuller, and R. Ramierez, Phys. Rev. B 43, 1843 (1991).

${ }^{13}$ P. Sigmund, Phys. Rev. 184, 383 (1969). 